\title{
Geographical and ethnic differences in Malassezia species distribution on healthy skin
}

\author{
Cheryl Leong, Joleen Goh, Antony Irudayaswamy, Thomas Dawson. \\ Skin Research Institute Singapore, Singapore
}

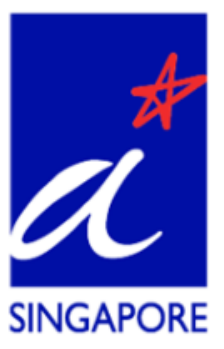

Agency for Science, Technology Skin Research Institute of Singapore

\section{INTRODUCTION}

Malassezia are lipid dependent yeasts which live as skin commensals. They are implicated in skin disease such as pityriasis versicolour and can cause serious opportunistic infection in immunocompromised individuals. Of the nine species found on human hosts, five $(M$. sympodialis, $M$. slooffiae, $M$. furfur, $M$. globosa, $M$. restricta) have been regularly isolated at varying frequencies (Figure 1, right). The factors accounting for these differences are unknown and could be due to culture method, climate or other factors.

Ethnic skin types vary in lipid content, hydration levels and skin barrier function, which may affect the local skin mycobiome. The tropical multi-racial demographics of Singapore is an ideal location for comparison of fungal ecology on different skin types.

In this study, we aimed to determine if skin ethnicity affects the species of Malassezia colonizing the skin of healthy individuals and if this may account for different species distributions observed worldwide.

\section{METHODS}

Malassezia were sampled from the skin of 40 healthy volunteers from 4 different ethnic backgrounds (Chinese, Malay, Indian and Caucasian) with equal gender distribution living in Singapore in accordance with our ethnical committee regulations. This involved swabbing of the nasal sidewall with a saline wetted swab and culture on modified Leeming-Notman agar for 7 days at $32^{\circ} \mathrm{C}$. Species identification of individual colonies was performed using molecular typing methods and ITS sequencing.

\section{RESULTS}

Culture-based characterization of a multi-racial Singapore cohort

- The average healthy individual in Singapore has 2-3 species of Malassezia on the skin.

- M. sympodialis, M. furfur, M. globosa and M. restricta were the most frequently cultured species respectively.

- Malassezia species distribution on healthy skin vary with ethnic group (Figure 2A \& B).

- The species composition within each ethnic group was similar between males and females.

Multi-locus strain typing of primary isolates suggest strain diversity is community and geography specific

- Comparisons of species data derived from different geographies (e.g. tropical vs temperate climate, different ethnicities) show Malassezia species diversity can be geography specific.

- Some community specific relationships can be traced (Figure 2C) based on Malassezia species diversity on the skin.

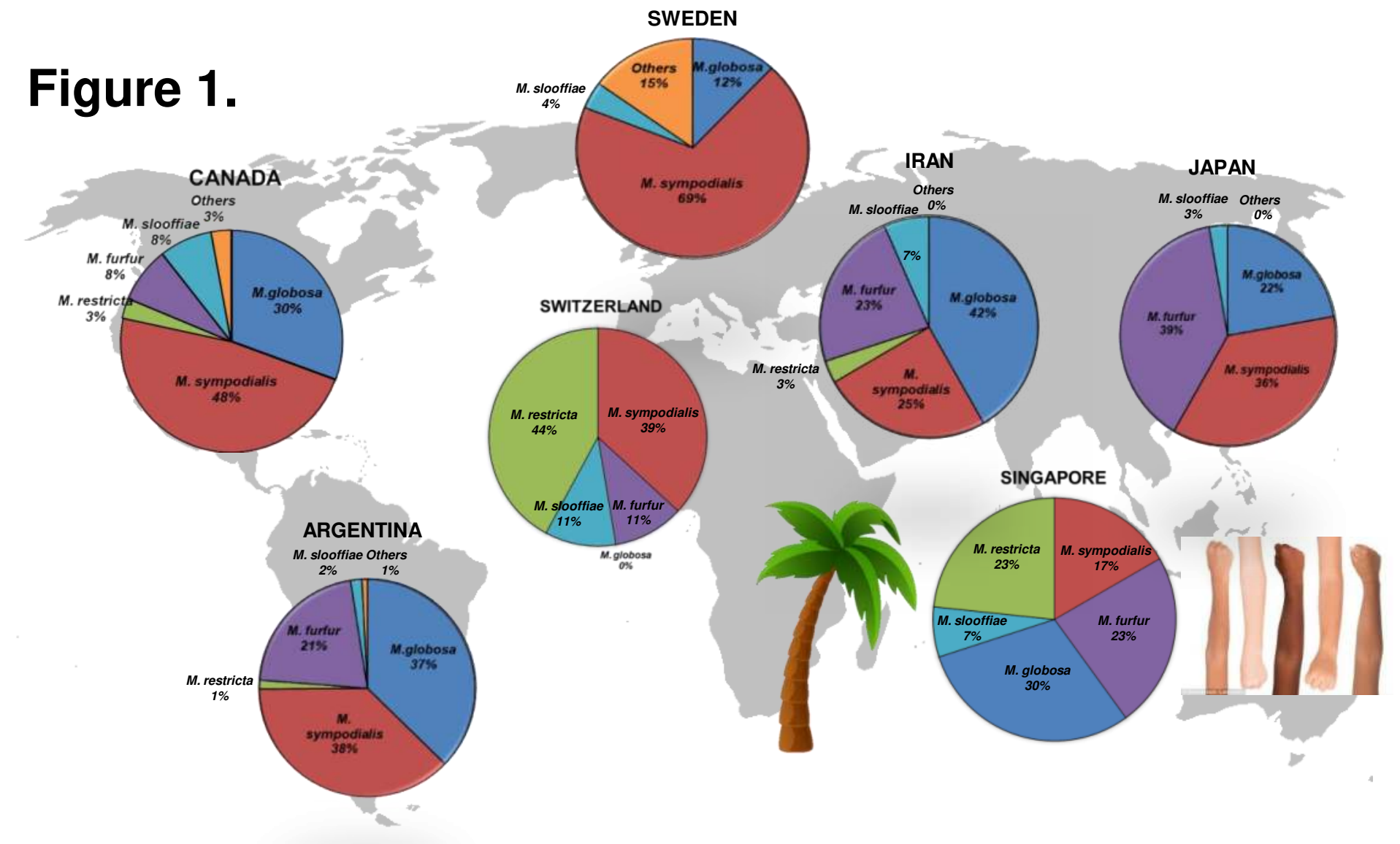

Figure 1. Pie charts showing the culture-based species distribution of Malassezia species derived from healthy individuals. Singapore is unique for its tropical climate and multi-ethnic demographics. ata adapted from Ashbee 2007. Switzerland and Singapore based data are authors own.

Figure 2.
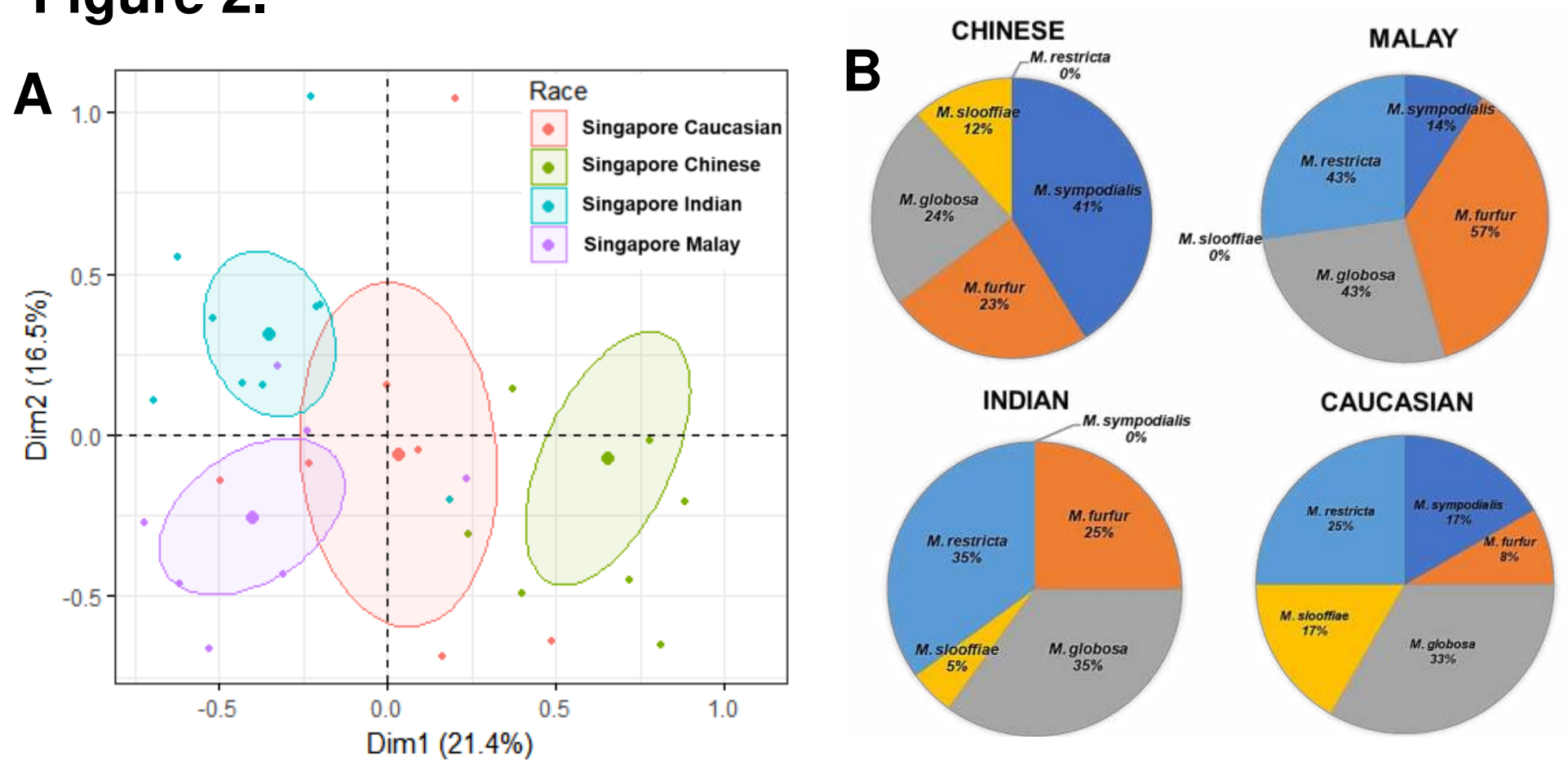

C Cluster Dendrogram

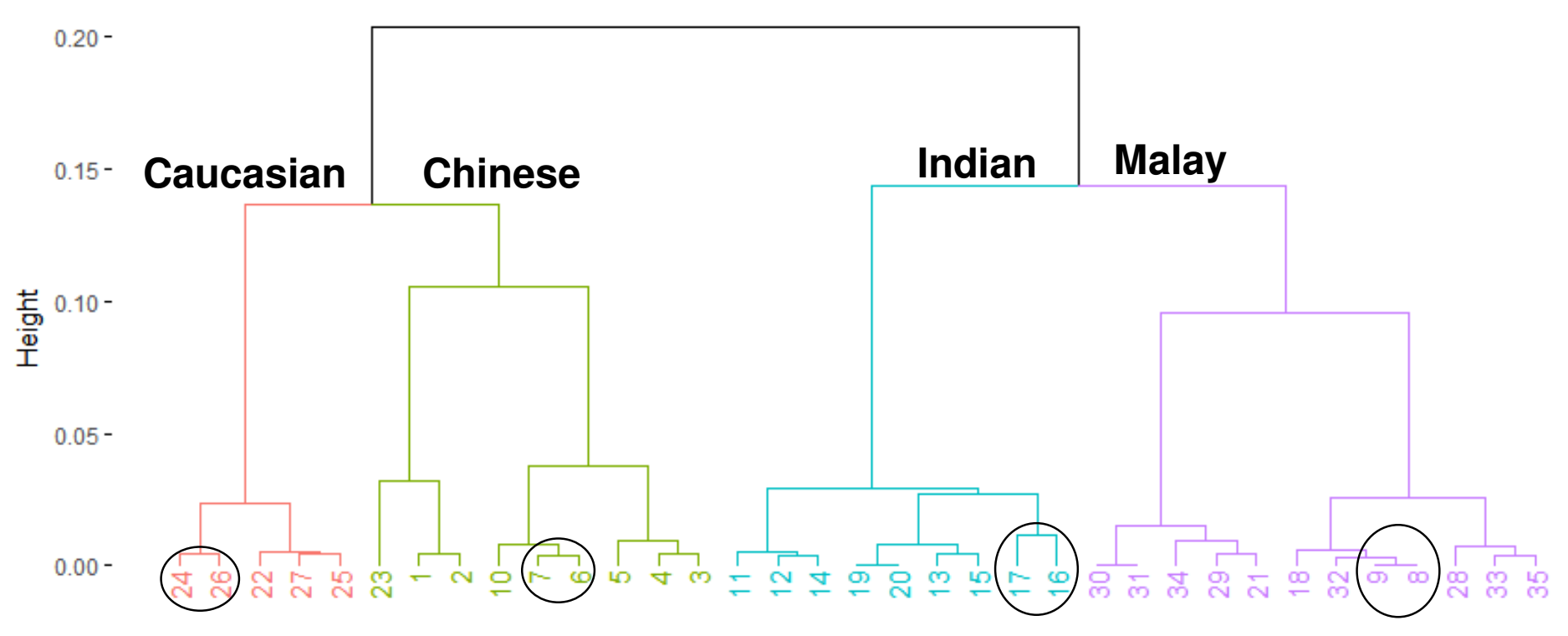

Figure 2(A). Multiple correspondence analysis of Malassezia species derived from four different ethnic groups in Singapore shows that individuals cluster according to their skin type, (B) five species of Malassezia are represented on the skin of Singaporeans although their proportions vary with ethnic group and (C) Cluster dendrogram analysis with community specific relationships delineated by the black circle.

\section{CONCLUSIONS}

Variations in were observed among different ethnic groups in Singapore. This suggests that skin ethnicity is a key underlying factor in the composition of the skin mycobiome, possible due to differential expression of lipid in different ethnic skin types.

It is likely that Malassezia studies around the world reflect a species distribution representative of the ethnic background of their local populations. Characterizing the healthy skin mycobiome composition and delineated geography and community specific strains will be useful in defining the role of Malassezia in health and disease. 